\title{
Small Bowel Obstruction in Patients with a Prior History of Cancer: Predictive Findings of Malignant Origins
}

\author{
Samir Johna
}

Published online: 19 October 2013

(C) Société Internationale de Chirurgie 2013

Surgeons occasionally have to answer some tough questions when it comes to managing small bowel obstruction (SBO) in patients with prior history of abdominal malignancy. In surgery, it is a fact of life to accept recurrent abdominal malignancy as bad news. The prognosis is grim at best, and most patients rarely live beyond 6-12 months once the diagnosis is made. Therefore, it makes sense to adopt less aggressive strategies for optimal management and compassionate care. On the other hand, non-malignant SBO deserves consideration for timely surgical intervention if we are to prevent potential morbidity and mortality. One may then ask: how can we distinguish between the two scenarios? Can we make a correct diagnosis relying on history and physical examination alone? What is the role of imaging, if any, that may help triage such patients?

There are no easy answers to these difficult questions. However, in this issue of the World Journal of Surgery, Douard et al. [1] provide us with a few solid tips based on over 1 decade of experience in management of such difficult conditions. The authors found that history holds some important clues. A sudden onset of unrelenting abdominal pain that is caused by high-grade SBO is less likely to be malignant, particularly if the obstruction occurs more than 3 years after the original abdominal operation. Clinical examination is of little value, if any. Imaging with abdominal computed tomography (CT) scan, while sensitive and specific for the diagnosis of bowel obstruction, is less accurate in identifying the etiology of the obstruction or in confirming the recurrence of the malignancy. Magnetic resonance imaging (MRI) is known to be much better. However, it is less available for around-the-clock use. The good news is that the authors also found SBO in patients with no known recurrent malignancy to be of benign origin in more than $70 \%$ of their patients. This is the cohort of patients that will probably benefit the most from an early surgical intervention when surgery is indicated.

So, where do we go from here? Based on the authors' experience, when managing these patients, surgeons should try to look for possible recurrent malignancy through careful history, basic physical examination, and appropriate imaging. One should probably start with abdominal CT scan and progress if needed to abdominal MRI when available. If there is no indication that a recurrent malignancy is the culprit for the SBO, surgeons should have a lower threshold to go to the operating room. Otherwise, patience and patience! Only fools rush in with a knife!

\section{Reference}

1. Douard R, Prost-à-la Denise J, Malamut G, et al Small bowel obstruction in patients with a prior history of cancer: predictive findings of malignant origins. World J Surg. doi:10.1007/s00268013-2303-3

\footnotetext{
S. Johna $(\bowtie)$

Loma Linda University School of Medicine, Loma Linda, CA,

USA

e-mail: samir.johna@gmail.com
} 